\title{
The Complete Decongestive Therapy to Treat Lymphedema in Post-Breast Cancer Surgery and Its Relationships with Patients Psychological and Physical Characteristics: A Pilot Study
}

\author{
Eva Mazzotti ${ }^{1}$, Roberto Bartoletti ${ }^{2}$, Claudia Sebastiani ${ }^{2}$, Alessandro Scoppola ${ }^{2}$, Paolo Marchetti ${ }^{2,3}$ \\ ${ }^{1}$ Department of Clinical and Molecular Medicine, Sant'Andrea Hospital, School of Medicine and Psychology, University "La Sapienza” \\ Rome, Italy \\ ${ }^{2}$ Division of Oncology and Oncological Dermatology- IDI-IRCCS, Rome, Italy \\ ${ }^{3}$ Department of Clinical Oncology, Sant'Andrea Hospital, School of Medicine and Psychology, University “La Sapienza” Rome, Italy
}

Email address:

eva.mazzotti@tiscali.it (E. Mazzotti)

\section{To cite this article:}

Eva Mazzotti, Roberto Bartoletti, Claudia Sebastiani, Alessandro Scoppola, Paolo Marchetti. The Complete Decongestive Therapy to Treat Lymphedema in Post-Breast Cancer Surgery and Its Relationships with Patients Psychological and Physical Characteristics: A Pilot Study. Journal of Cancer Treatment and Research. Vol. 3, No. 3, 2014, pp. 37-41. doi: 10.11648/j.jctr.20150303.13

\begin{abstract}
Background. More than $20 \%$ of women treated for breast cancer are at risk for developing lymphedema, a chronic condition that causes swelling, pain, altered appearance and reduced mobility. Complete Decongestive Therapy (CDT) is considered the gold standard to treat lymphedema. Objective. To measure lymphedema features and psychological variables in 10 post-breast cancer surgery patients at baseline, and 1, 6, and 12 months after CDT. Results. Lymphedema appeared between 0 and 6 years after breast cancer surgery. After 4 weeks in CDT treatment, the median degree of reduction was 38.73\% (range, 7.45-58.39), and six months after, 49.57\% (range, 11.91-82.50). Comparing patients that have had an extra reduction in arm circumferences at T3 (group 1) and patients that did not (group 2), we found: patients in group 1 showed at baseline a better psychological functioning (health-related quality of life domains, anxiety, depression, body-image vulnerability, appearance stereotyping), and higher scores in curability and severity respect to the comparison group. 12 months after the CDT, among patients of the group 1, the lymphedema is perceived as less severe and more curable compared to their perception at baseline, and dysfunctional investment in one's appearance and anxiety scores decreased, emotional wellbeing improved. Patients in the group 2 perceived lymphedema as much more curable, but also much more severe that at baseline. Both, anxiety and dysfunctional investment in one's appearance scores increased. Conclusions. Too often, conventional rehabilitation may not optimally match clinical resources to patients' needs, and if our results will be confirmed, screening and independent treatment for psychological distress will be required in patients to prevent poor CDT responses.
\end{abstract}

Keywords: Breast Cancer, Complete Decongestive Therapy, Lymphedema, Follow up Study, Post-Breast Cancer Surgery Effect

\section{Introduction}

Lymphedema, also known as lymphatic obstruction, is a condition of localized fluid retention and tissue swelling caused by a compromised lymphatic system. Lymphedema may be inherited (primary) or caused by injury to the lymphatic vessels (secondary). In women, it is most prevalent in the upper limbs after breast cancer surgery, in particular after axillaries lymph node dissection, ${ }^{1}$ occurring in the arm on the side of the body in which the surgery is performed. In Western countries, secondary lymphedema is most commonly due to cancer treatment. ${ }^{2}$ Between 38 and $89 \%$ of breast cancer patients suffer from lymphedema due to axillary lymph node dissection, surgery and/or radiation therapy. $1,3-7$

In many patients with cancer, this condition does not develop until months or even years after therapy has concluded. 
The diagnosis or early detection of lymphedema is difficult. The first signs may be subjective observations such as "my arm feels heavy" or "I have difficulty these days getting rings on and off my fingers". These may be symptomatic of early stage of lymphedema where accumulation of lymph is mild and not detectable by any difference in arm volume or circumference. As lymphedema develops further, definitive diagnosis is commonly based upon an objective measurement of differences between the affected or at-risk limb at the opposite unaffected limb, e.g. in volume or circumference.

Lymphedema develops in stages, from mild to severe.

The most common method of staging was:

Stage 0 (latent): The lymphatic vessels have sustained some damage which is not yet apparent. Transport capacity is still sufficient for the amount of lymph being removed. Lymphedema is not present.

Stage 1 (spontaneously reversible): Tissue is still at the pitting stage: when pressed by the fingertips, the affected area indents, and reverses with elevation. Usually upon waking in the morning, the limb or affected area is normal or almost normal in size.

Stage 2 (spontaneously irreversible): The tissue now has a spongy consistency and is considered non-pitting: when pressed by the fingertips, the affected area bounces back without indentation. Fibrosis found in stage 2 lymphedema marks the beginning of the hardening of the limbs and increasing size.

Stage 3 (lymphostatic elephantiasis): At this stage, the swelling is irreversible and usually the $\operatorname{limb}(\mathrm{s})$ or affected area is very large. The tissue is hard (fibrotic) and unresponsive; some patients consider undergoing reconstructive surgery, called "debulking". This remains controversial, however, since the risks may outweigh the benefits, and the further damage done to the lymphatic system may in fact make the lymphedema worse.

Though incurable and progressive, a number of treatments can ameliorate symptoms.

Lymphedema is an important consideration for clinicians because of its relatively high frequency and effect on survival, and quality of life. ${ }^{8-10}$

The patterns of lymphedema evolution could be different among patients, ${ }^{11}$ ranging widely in severity, but specific early treatments could reduce its evolution. ${ }^{12-15}$ Complete Decongestive Therapy (CDT), a two-phase program that consists of a treatment phase and a maintenance phase, is considered the gold standard of treatment for lymphedema that has progressed beyond Stage I. ${ }^{16}$

Aim of this study was to measure lymphedema features and psychological variables in post-breast cancer surgery at baseline, 1, 6, and 12 months after CDT.

\section{Methods}

A longitudinal study employing clinical assessments followed patients over 13 months to assess issues related to arm morbidity post-breast cancer surgery. Arm circumference and volume were used to measure upper limb impairments. Health-Related Quality of Life (HRQOL) was rated using the Functional Assessment of Cancer Treatment-General scale (FACT-G) ${ }^{17}$ and the FACT-Breast cancer subscale (FACT-B+4). ${ }^{18}$ Anxiety and depression were rated using the Hospital Anxiety and Depression Scale (HADS). ${ }^{19}$ Dysfunctional investment in one's appearance was measured with the Appearance Schemas Inventory (ASI). ${ }^{20}$ Three visual analogue scales (VASs) were used to quantify patients' perception of lymphedema severity and curability, and quality of life. ${ }^{21}$

The circumferences of the study patient's arm were measured at fixed points along the arm, using a marking board. At the initial visit, and subsequently at each visit of follow up, the arm was marked and measured at the same fixed points, usually $5 \mathrm{~cm}$ intervals. The same operator (RB) measured the patient at each visit and measurements were made without prior reference to previous results. The contralateral arm was measured at the initial visit for all patients and it was measured at all subsequent visits of follow up (excepted at T1).

Also water displacement volume measurements were used. Adjustments for right or left handedness were not made.

A difference of $2.0 \mathrm{~cm}$ between the affected and unaffected arm has been used to indicate a positive diagnosis of lymphedema. ${ }^{15}$

Inclusion criteria for this study were: female gender, age more than 18 years, breast cancer surgery with axillaries lymph node dissection, chemotherapy and radiotherapy completed, unilateral secondary post-breast cancer surgery lymphedema, CDT approved by oncologist, life expectancy greater than 1 year, written informed consent. Exclusion criteria were: patients already treated with CDT (previous six months), patients affected by some medical conditions, including acute phlebitis or thrombo-phlebitis, lymphangitis and/or erysipelas, peripheral neuropathy in the arm affected (deemed by the investigator to be likely to interfere with participation and compliance with the study procedure), patients that cannot guarantee regular follow-up visits for logistic or geographical reasons.

The right of the patient to withdraw from the study in any time, without giving reasons has been reported in the consent form. Before testing, informed consent was obtained from each subject, and the study was approved by the Hospital Ethics Committee.

Physical and psychological measures were collected at baseline (T0), after 4 weeks of CDT (T1), 6 (T2) and twelve months (T3) after the end of the CDT.

Ten women with unilateral secondary post-breast cancer surgery lymphedema, either new cases or already in followup at the IDI-IRCCS Division of Oncology, participated in the pilot study. 


\section{Data Reduction and Statistical Analyses}

For descriptive purposes the study variables were categorized: subjects were subdivided into two groups with respect to years of education $(<=13,>13)$, marriage status (no/yes). Lymphedema was graded using the International Lymphology Society grading system. ${ }^{13}$

The severity of lymphedema at baseline (T0) was calculated by reference to the contralateral arm (unaffected) in each patient. At each visit of follow up the circumferences (and volume) at each marked point on the affected arm were summed, as also in the contralateral arm (except at T1). The sum of the circumferences at each visit following the commencement of treatment (T1-T3) was compared with the sum of the circumferences at the visit before. The initial percentage degree of enlargement was estimated as: ((baseline affected arm - baseline contralateral arm)/ baseline affected arm) x100.

The simplest estimate of the effect of treatment was the percentage reduction in sum of the circumferential (or volume) measurements of the affected arm at each visit of follow up.

Lymphedema improvement was defined as a reduction of more than 3 percent points from baseline total circumference.

In this study we presented the measures and the effects of CDT for all the variables in study at T1 (short-time effect) and T3 (long-time effect).

Statistical analysis was conducted using STATA 11.

\section{Results}

Baseline. Patients mean age was 64 (standard deviation SD, 10.6; range 43-75), 60\% were married and 70\% had one or two children; $60 \%$ had more than 13 years of school. Mean BMI was 24.8 (SD, 2.0; range 21.3-27.7).

Fifty percent of patients have had a radical surgery (mastectomia) and the mean axillaries lymph nodes removed was 18.2 (SD, 6.2; range 6-27). All of them were treated with chemotherapy and hormone-therapy, and 90\% were also treated with radiotherapy.

Lymphedema appeared 0-6-years after breast cancer surgery (mean, 2.4; SD, 1.9). In 40\% it appeared in the first year, in $40 \%$ between 1 and 5 years, and in $20 \%$ between 5 and 6 years after. It appeared from 50 to 935 days before the inclusion in the study (median length, 375 days), and for $40 \%$ of patient it affected the left arm.

Lymphedema was in stage II, also called "spontaneously irreversible lymphedema" (40\%) or in stage III, also called "lymph static elephantiasis" (60\%).

At baseline (T0), mean difference in total arm circumferences between affected and non-affected arm was
$35.8 \mathrm{~cm}(\mathrm{SD}, 18.5$; range, 16.7-68.0 cm; median affected arm circumference $255.65 \mathrm{~cm}$ and contralateral arm circumference 224.30). The median degree of enlargement was 15.6 per cent (range, 6.12-27.68). Differences between 'normal-weight' patients $(\mathrm{BMI}<25 ; 60 \%)$ and 'over-weight' patients $(40 \%)$, or between right or left affected arm were not apparent.

Follow-up. Patient compliance during the treatment program was good. All 10 patients returned for regular reviews at 1, 6, and 12 months. Specific symptoms became a-specific (86\%), and hand, forearm and arm involvement improved, resulting in a reduction in disability (75\%).

At $\mathrm{T} 1$, the median total affected arm circumference was $240.05 \mathrm{~cm}$ (contralateral arm, not registered). At T2, was $238.21 \mathrm{~cm}$ (contralateral arm, 224.50). At T3, was 239.90 $\mathrm{cm}$ (contralateral arm, 224.50).

After 4 weeks in CDT treatment (T1), the median degree of reduction was $38.73 \%$ (range, 7.45-58.39), and six months after (T2), the median degree of reduction was 49.57\% (range, 11.91-82.50).

Follow up at 12 months (T3) shows a median degree of reduction of $0.41 \%$ (range $-1.63-9.48$ ). Ten per cent of patients get worse, $40 \%$ were stable and $50 \%$ showed a socalled 'extra-reduction'. Differently, a clinical significant decrement in circumference occurred in $70 \%$ of patients after four weeks of CDT (defined as a reduction of more than 3 percent points from baseline total circumference).

In the whole sample, no effects appeared in psychological distress and quality of life scores, except for curability (patients judge lymphedema more curable than before CDT) and severity (patients judge lymphedema less severe than before CDT).

In Table 1, psychological characteristics mean scores (and SDs), separately for patients that have had an extra reduction in arm circumferences at $\mathrm{T} 3$ and patients that did not, and separately for time of follow up, are shown. Comparing the two groups we found: (1) patients that have had an extra reduction at T3, showed at baseline a better psychological functioning (health-related quality of life domains, anxiety, depression, body-image vulnerability, appearance stereotyping), and higher scores in curability and severity respect to the comparison group. (2) 12 months after the CDT, among patients that have experienced an improvement, the lymphedema is perceived as less severe and more curable compared to their perception at baseline, the dysfunctional investment in one's appearance and anxiety decreased, while emotional wellbeing improved. Patients in the comparison group perceived lymphedema as much more curable, but also much more severe that at baseline. Both, anxiety and dysfunctional investment in one's appearance increased. 
Table 1. Patients' perceived lymphedema severity and curability and quality of life (PI-SCQ), health-related quality of life as measured by FACT-G and FACT$B$ scales, anxiety and depression as measured by HADS, body image vulnerability, self investment, and appearance stereotyping as measured by ASI at T0, TI, and T3, separately for patients that have had an extra reduction in arm circumferences from $T 1$ and $T 3(N=5)$, and patients that did not (N=5) (means and standard deviations).

\begin{tabular}{lllllll}
\hline & \multicolumn{2}{l}{ Pts with extra reduction $(>\mathbf{3} \%)$} & \multicolumn{3}{l}{ Pts without extra reduction } \\
\hline & T0 & T1 & T3 & T0 & T1 & T3 \\
\hline PI-SCQ & mean (sd) & mean (sd) & mean (sd) & mean (sd) & mean (sd) & mean (sd) \\
severity & $8.0(1.2)$ & $7.3(1.2)$ & $7.3(1.0)$ & $5.2(0.5)$ & $7.3(2.2)$ & $7.3(2.2)$ \\
curability & $7.5(2.0)$ & $8.3(2.1)$ & $8.8(1.5)$ & $5.5(1.9)$ & $7.8(1.7)$ & $8.0(1.8)$ \\
quality of life & $7.3(1.5)$ & $7.5(3.0)$ & $8.3(2.1)$ & $7.3(2.2)$ & $7.6(1.7)$ & $6.8(2.5)$ \\
FACT-G & & & & & \\
physical wellbeing & $90.2(10.7)$ & $92.9(6.5)$ & $94.6(6.2)$ & $80.4(22.5)$ & $88.1(2.1)$ & $83.0(15.3)$ \\
soc.-familial wellbeing & $69.6(4.6)$ & $61.6(11.1)$ & $63.4(16.1)$ & $68.8(7.9)$ & $66.1(11.1)$ & $61.6(5.4)$ \\
emotional wellbeing & $71.9(20.2)$ & $81.3(14.4)$ & $82.3(11.5)$ & $69.8(17.5)$ & $71.9(9.2)$ & $66.7(9.0)$ \\
functional wellbeing & $68.8(19.2)$ & $58.9(16.1)$ & $61.6(9.8)$ & $53.6(5.0)$ & $60.7(15.7)$ & $53.6(7.7)$ \\
FACT-B & $52.1(26.7)$ & $58.3(15.9)$ & $61.1(9.6)$ & $47.2(9.9)$ & $46.5(11.2)$ & $50.7(12.7)$ \\
HADS & & & & & \\
anxiety & $42.9(16.5)$ & $45.2(16.7)$ & $33.3(19.5)$ & $47.6(17.8)$ & $56.0(9.8)$ & $56.0(18.0)$ \\
depression & $28.6(17.0)$ & $29.8(9.0)$ & $30.9(8.3)$ & $42.9(15.6)$ & $40.5(14.8)$ & $42.9(23.3)$ \\
ASI & & & & & \\
BIV & $32.3(20.8)$ & $26.0(12.1)$ & $25.0(14.4)$ & $42.7(8.7)$ & $38.6(15.2)$ & $43.1(10.5)$ \\
SI & $60.0(28.6)$ & $47.5(20.2)$ & $41.3(25.0)$ & $57.5(24,0)$ & $57.5(16.6)$ & $66.3(17.0)$ \\
AS & $39.6(27.5)$ & $29.2(24.1)$ & $33.3(7.0)$ & $54.2(30.8)$ & $45.8(17.4)$ & $52.1(11.5)$ \\
\hline
\end{tabular}

$\mathrm{T} 0=$ baseline; $\mathrm{T} 1=4$-weeks-CDT; $\mathrm{T} 3=12$-months of follow up

$\mathrm{BIV}=$ body image vulnerability; $\mathrm{SI}=$ self investment; $\mathrm{AS}=$ appearance stereotyping

\section{Discussion}

Several changes in lymphedema clinical characteristics at short-, medium, and long-term emerged from our study. At short (T1) and long-term (T3) positive effects emerged in arm circumference reduction (as in volume reduction, data not shown).

On the whole sample, no effects appeared in psychological distress and quality of life scores, excepted for perception of curability and severity. In contrast, comparing patients that at the end of the study reported an extra reduction in the lymphedema severity with the other that did not, several interesting results emerged. The long-term expected effects seem to be associated with a better psychological status at baseline.

If we take into account the chronic and remarkable trend versus spontaneous evolution of the lymphatic pathology secondary to lymphectomia, it is important to pay attention to the necessity to have a shared instrumental-clinical approach with a double objective. It is crucial to intercept patients showing the very early symptoms of lymphatic illness as well those displaying clinically advanced lymphedema. This is an efficient way to offer these patients personalized rehabilitative and integrated treatment in which different professional figures, in respect to their reciprocal competencies, can give appropriate answers at a physical, functional, and psychological level. In accord to a biopsychosocial perspective the interventions for cancer and symptom management could be the most efficient treatments to understanding and managing patient's needs. In this prospective the possibility of individually tailoring treatment for each patient is increased as that of better outcomes.
The small sample size did not allow us to evaluate the role of other variables in the relationship between psychological status at baseline and lymphedema improvement. However, if these results will be confirmed in other study, become of great importance to embed the psychological assessment in the baseline clinical evaluation for secondary post-breast cancer surgery lymphedema.

\section{Conclusions}

These results suggest that physical rehabilitation is required for lymphedema to improve short and long-term post-operative physical functioning. A relationships emerged between a satisfactory functioning in several psychological variables at baseline and an extra reduction in lymphedema measures 12 months later in patients treated with CDT.

Too often, conventional rehabilitation may not optimally match clinical resources to patients' needs. If these results will be confirmed, screening and independent treatment for psychological distress will be required in patients to prevent poor CDT responses.

\section{References}

[1] Burt J, White G. Lymphedema: a breast cancer patient's guide to prevention and healing. Hunter House. p. 9. 2005. ISBN 978-0-89793-458-9

[2] Brorson H, Ohlin K, Olsson G, Svensson B, Svensson H. Controlled compression and liposuction treatment for lower extremity lymphedema. Lymphology 2008; 41(2):52-63. PMID 18720912 
[3] Kissin MW, Querci della Rovere G, Easton D, Westbury G. Risk of lymphoedema following the treatment of breast cancer. $\quad \mathrm{Br}$ J Surg 1986;73(7):580-584. doi: 10.102/bjs.1800730723. PMID 3730795

[4] Segerström K, Bjerle P, Graffman S, Nyström A. Factors that influence the incidence of brachial oedema after treatment of breast cancer. Scand J Plast Reconstr Surg Hand Surg 1992;26(2):223-227. doi:10.3109/02844319209016016. PMID 1411352

[5] Hayes SC, Johansson K, Stout NL, et al. Upper-body morbidity after breast cancer: incidence and evidence for evaluation, prevention, and management within a prospective surveillance model of care. Cancer 2012;118:2237-22doi: $10.1002 /$ cncr 27467

[6] Tasj RJ, Dennis LK, Lynch CF, Snetselaar LG, Zamba GK, Scott-Conner C. The Risk of developing arm lymphedema among breast cancer survivors: a meta-analysis of treatment factors. Ann Surg Surg Oncol. 2009;16:1959-1972

[7] Di Sipio T, Rye S, Newman B, Hayes S. Incidence of unilateral arm lymphedema after breast cancer: a systematic review and meta-analysis. Lancet Oncol 2013;14(6):5005doi:10.1016/S1470-2045(13)70076-7

[8] Hayes S, Di Sipio T, Rye S, et al. Prevalence and Prognostic Significance of Secondary Lymphedema Following Breast Cancer. Lymphatic Research and Biology 2011;9(3):1351doi:10.1089/1rb.2011.0007

[9] Ahmed RL, Prizment A, Lazovich D, Schmitz KH, Folsom AR. Lymphedema and quality of life in breast cancer survivors: the Iowa Women's Health Study. J Clin Oncol 2008;26(35):5689-5696

[10] Kwan W, Jackson J, Weir LM, Dingee C, McGregor G, Olivotto IA. Chronic arm morbidity after curative breast cancer treatment: prevalence and impact on quality of life. $J$ Clin Oncol 2002;20(20):4242-4248

[11] Norman SA, Localio AR , Potashnik SL et al. Lymphedema in breast cancer survivors: incidence, degree, time course, treatment, and symptoms. J Clin Oncol 2009;27( 3):390-397
[12] Lawenda BD, Mondry TE, Johnstone Peter AS. Lymphedema: a primer on the identification and management of a chronic condition in oncologic treatment. CA Cancer $J$ Clin 2009;59:8-24

[13] International Society of Lymphology. The diagnosis and treatment of peripheral lymphedema. Consensus document of the International Society of Lymphology. Lymphology 2003;36:84-91

[14] Armer JM, Stewart BR, Shook RP. 30-month post Breast cancer Treatment Lymphedema. Journal of Lymphology 2009;4(1):14-18

[15] Hayes SC, Janda M, Cornish B, Battistutta D, Newman B. Lymphedema After Breast Cancer: Incidence, Risk Factors, and Effect on Upper Body Function. J Clin Oncol 2008;26:3536-3542

[16] Huang TW, Tseng SH, Lin CC, et al. Effects of manual lymphatic drainage on breast cancer-related lymph edema: a systematic review and meta-analysis of randomized controllated trials. World J Surgical Oncology 2013;11:15

[17] Cella DF, Tulsky DS, Gray G, et al. The Functional Assessment of Cancer Therapy (FACT) scale: Development and validation of the general measure. $J$ Clin Oncol 1993;11:570-579

[18] Coster S, Poole K, Fallowfield L. The validation of a quality of life scale to assess the impact of arm morbidity in breast cancer patients post-operatively. Breast Cancer Research and Treatment 2001;68:273-282

[19] Bjelland I, Dahl AA, Haug TT, Neckelmann D. The validity of the Hospital Anxiety and Depression Scale. An update literature review. J Psychosom Res 2002;52:69-77

[20] Cash TF, Labarge AS. Development of the Appearance Schemas Inventory: A new cognitive body-image assessment. Cognitive Therapy and Research 1996;20:37-50

[21] Mazzotti E, Sebastiani C, Marchetti P. Patient-perception of the disease control and relationship with psychosocial variables. Cancer Management and Research 2012;4:3353doi: 10.2147/CMAR.S35060 